\title{
Particle emission following Coulomb excitation in ultrarelativistic heavy-ion collisions
}

\author{
I.A. Pshenichnov ${ }^{1,2, \star}$, I.N. Mishustin ${ }^{2,3}$, J.P. Bondorf $f^{2, \dagger}$, \\ A.S. Botvina ${ }^{1,4}$, A.S. Iljinov ${ }^{1}$ \\ ${ }^{1}$ Institute for Nuclear Research, Russian Academy of Science, \\ 117312 Moscow, Russia \\ 2 Niels Bohr Institute, DK-2100 Copenhagen, Denmark \\ ${ }^{3}$ Kurchatov Institute, Russian Research Center, \\ 123182 Moscow, Russia \\ 4 Dipartimento di Fisica and INFN, 40126 Bologna, Italy
}

\begin{abstract}
We study nuclear reactions induced by virtual photons associated with Lorentz-boosted Coulomb fields of ultrarelativistic heavy ions. Evaporation, fission and multifragmentation mechanisms are included in a new RELDIS code, which describes the deexcitation of residual nuclei formed after single and double photon absorption in peripheral heavy-ion collisions. Partial cross sections for different dissociation channels, including the multiple neutron emission ones, are calculated and compared with data when available. Rapidity and transverse momentum distributions of nucleons, nuclear fragments and pions, produced electromagnetically, are also calculated. These results provide important information for designing large-rapidity detectors and zero-degree calorimeters at RHIC and LHC. The electromagnetic dissociation of nuclei imposes some constrains on the investigation of exotic particle production in $\gamma \gamma$ fusion reactions.
\end{abstract}

PACS: 25.75.-q, 25.20.-x, 25.70.De, 24.10.Lx

Key words: ultrarelativistic heavy ions, photonuclear reactions, Coulomb excitation, intranuclear cascade model, statistical multifragmentation

^ E-mail: pshenichnov@al10.inr.troitsk.ru † E-mail: bondorf@nbi.dk 


\section{Introduction}

There exist many mechanisms of particle production, nucleon and fragment emission in nuclear collisions at ultrarelativistic energies. They can be divided into the following ample categories. The first category contains collisions with direct nuclear overlap. In addition to nucleon-nucleon or parton-parton collisions, which are the most obvious sources of secondary hadrons, collective mechanisms were considered for baryon-antibaryon pair [1] or dilepton [2] production. The second category deals with "distant" electromagnetic collisions without nuclear overlap. In such a situation the interaction can be treated in terms of virtual photons emitted by one nucleus and absorbed by another collision partner. A subsequent photonuclear $(\gamma A)$ reaction leads to a so-called Electromagnetic Dissociation (ED) of nuclei [3, 4]. The virtual photon flux and consequently the rate of such $\gamma A$ events is proportional to $Z^{2}$, where $\mathrm{Z}$ is the charge of the first nucleus. The ED process is widely used for studying the properties of Giant Resonances (GR) in nuclei, including multiphonon excitations [1].

In Ref. [5] the description of nuclear photoabsorption was extended to the photon energies much above the GR region, where the exitation of individual nucleons and multiple pion production become important. The model of electromagnetic dissociation processes taking into account these high-energy photon absorption channels was constructed in Ref. [6]. According to this model, the fast hadrons produced after the photon absorption initiate a cascade of subsequent collisions with the intranuclear nucleons leading to the heating of a residual nucleus. At a later stage the nucleus undergoes de-excitation by means of the evaporation of nucleons and lightest fragments, binary fission or multifragmentation. The latter process takes place when the excitation energy is comparable with the total binding energy of a residual nucleus [7].

Another interesting mechanism in the second category is the photon-photon collision or $\gamma \gamma$ fusion leading to the particle production at mid-rapidity [8, 9, 10]. The rate of these processes is proportional to $Z^{4}$. The effective $\gamma \gamma$ luminosity at the LHC collider is expected to be comparable with that at a possible future Photon Linear Collider [11].

It is worthwhile to mention that the processes of the second category have attracted a special attention in the last few years. This interest is generated by the construction of the new heavy-ion colliders: RHIC at Brookhaven $(\mathrm{Au}+\mathrm{Au}$ with $\sqrt{s}=200 \mathrm{GeV})$ and LHC at CERN $(\mathrm{Pb}+\mathrm{Pb}$ with $\sqrt{s}=5500 \mathrm{GeV})$. It is expected that the ED processes will play an important role for these energies and especially for the ions with high $Z$, due to the above-mentioned $Z^{2}$-dependence. The predicted cross section for the electromagnetic dissociation at LHC, $\sigma_{E D}$, is so large $(\approx 200 \mathrm{~b})$ that together with the electron capture process $(\approx 100 \mathrm{~b})$, it will reduce significantly the life time of relativistic heavy-ion beams [12] as compared with the proton ones. The ED reaction rate at LHC is expected to be

30 times higher than that for the nuclear interactions $\left(\sigma_{n u c} \approx 7 \mathrm{~b}\right)$. But of course 
the ED reactions are much less violent then the nuclear ones.

Hadron production in photon-photon and photon-pomeron collisions, as well as in double-pomeron exchange were studied in Ref. [13]. All these reactions are characterised by a large rapidity gap separating the remnants of the colliding hadrons or heavy ions from the particles produced in the central rapidity region. High-mass diffraction reactions were simulated by means of the Monte-Carlo event generator Phojet [14]. On the other hand, there are no "ready-to-use" event generators for simulating relatively "soft" $\gamma A$ processes, where nuclear degrees of freedom play an important role. In particular, such simulations are needed for estimating the probabilities of different disintegration channels of the colliding nuclei. The properties of the ED processes have to be well understood in order to distinguish them from very peripheral nuclear collisions.

In the present paper we consider the whole set of mechanisms of Coulomb excitation and subsequent dissociation of relativistic heavy-ions (RHI). In Sec.2 the model is presented and compared with existing experimental data on few neutron removal from $\mathrm{Au}$ and $\mathrm{Pb}$ nuclei by virtual and real photons. In Sec.3 we discuss the ED processes which are expected to be important at the future heavyion colliders, RHIC and LHC. We present the model predictions for multiplicity and transverse momentum distributions of neutrons. These results are important for designing zero-degree calorimeters for beam luminosity monitoring. Rapidity and transverse momentum distributions of $\pi^{+}$and $\pi^{-}$mesons, fission products and intermediate mass fragments are also discussed in Sec.3. Sec.4 is reserved for discussion and conclusions.

\section{Model of electromagnetic dissociation of heavy nuclei}

\subsection{Single- and double-photon absorption}

Let us consider the Coulomb excitation of an ultrarelativistic projectile. According to the Weizsäcker-Williams (WW) method the spectrum of virtual photons from a stationary target of charge $Z_{t}$ as seen by a projectile moving with velocity $\beta=v / c$ at impact parameter $b$ is expressed as [15]:

$$
N\left(E_{\gamma}, b\right)=\frac{\alpha Z_{t}^{2}}{\pi^{2}} \frac{\mathrm{x}^{2}}{\beta^{2} E_{\gamma} b^{2}}\left(K_{1}^{2}(\mathrm{x})+\frac{1}{\gamma^{2}} K_{0}^{2}(\mathrm{x})\right)
$$

where $\alpha$ is the fine structure constant, $K_{0}$ and $K_{1}$ are the modified Bessel functions of zero and first order, $\mathbf{x}=E_{\gamma} b /(\gamma \beta \hbar c)$. It is worth noting that the distribution (1) is written in the projectile rest frame. This means that for colliding beams one should use the Lorentz factor of one collision partner in the rest frame of another partner, $\gamma=2 \gamma_{\text {beam }}^{2}-1$, where $\gamma_{\text {beam }}$ is the Lorentz factor of each beam. 
The projectile can be exited by absorbing one or more virtual photons. Multiple excitations of the giant dipole resonance (GDR) up to the fourth order were considered in Refs. [1, 16]. Since the $n t h$-order cross section behaves approximately as $\left(\alpha Z_{t}^{2}\right)^{n}$, one should expect the maximum effect for the excitation of ${ }^{238} \mathrm{U}$ projectile in the field of ${ }^{238} \mathrm{U}$ target at ultrarelativistic energies. As was found in Ref. [4], even in this case the cross sections of the third and fourth order processes are quite small in comparison with that for the second order excitation: $\sigma^{(3)} / \sigma^{(2)} \approx 0.11$ and $\sigma^{(4)} / \sigma^{(2)} \approx 0.01$. Thus we restrict our consideration by the first and second order processes.

The mean number of photons absorbed by the projectile of mass $A_{p}$ in a collision at impact parameter $b$ is defined by

$$
m(b)=\int_{E_{\min }}^{\infty} N\left(E_{\gamma}, b\right) \sigma_{A_{p}}\left(E_{\gamma}\right) d E_{\gamma}
$$

where $\sigma_{A_{p}}\left(E_{\gamma}\right)$ is the appropriate photoabsorption cross section, either measured for this projectile nucleus with real photons or calculated within a model. For the cases of interest, i.e. for $\mathrm{Au}$ and $\mathrm{Pb}$ nuclei, we use the data set published in Refs. 117, 18, 19, 20], including a most recent one 21] where corresponding photoabsorption cross sections were measured. In the GDR region one can use a set of Lorentz-line approximations given in the review [22]. According to prescriptions of Ref. [20], the photoabsorption cross sections obtained in Refs. [17] and [19] for $\mathrm{Au}$ and $\mathrm{Pb}$ nuclei are rescaled by factors 0.93 and 1.22, respectively.

Following Llope and Braun-Munzinger [16] we assume that at an impact parameter $b$ the probability of the multi-photon absorption is given by the Poisson distribution with the mean multiplicity $m(b)$ defined by Eq. (2). Then the probability for absorbing exactly one photon of any energy during a collision at impact parameter $b$ is equal to:

$$
P^{(1)}(b)=m(b) e^{-m(b)},
$$

and the probability for absorbing exactly two photons is:

$$
P^{(2)}(b)=\frac{m^{2}(b)}{2 !} e^{-m(b)} .
$$

The normalized probability density that in a collision at impact parameter $b$ the absorbed photon has an energy $E_{1}$ is given by:

$$
q^{(1)}\left(E_{1}, b\right)=\frac{N\left(E_{1}, b\right) \sigma_{A_{p}}\left(E_{1}\right)}{m(b)} .
$$

Analogously, the probability density that in a second order process two photons have energies $E_{1}$ and $E_{2}$ is:

$$
q^{(2)}\left(E_{1}, E_{2}, b\right)=\frac{N\left(E_{1}, b\right) \sigma_{A_{p}}\left(E_{1}\right) N\left(E_{2}, b\right) \sigma_{A_{p}}\left(E_{2}\right)}{m^{2}(b)} .
$$


To consider particular ED channels i.e. neutron emission, fission, multifragmentation or pion production, which are studied in the present paper, we define the differential cross sections for the first and second order processes as:

$$
\frac{d \sigma_{i}^{(1)}}{d E_{1}}=2 \pi \int_{b_{\min }}^{\infty} b d b P^{(1)}(b) q^{(1)}\left(E_{1}, b\right) f_{i}^{(1)}\left(E_{1}\right)
$$

and

$$
\frac{d^{2} \sigma_{i}^{(2)}}{d E_{1} d E_{2}}=2 \pi \int_{b_{\min }}^{\infty} b d b P^{(2)}(b) q^{(2)}\left(E_{1}, E_{2}, b\right) f_{i}^{(2)}\left(E_{1}, E_{2}\right)
$$

Here $f_{i}^{(1)}\left(E_{1}\right)$ and $f_{i}^{(2)}\left(E_{1}, E_{2}\right)$ are the branching ratios for the considered channel $i$.

Finally, the integral cross sections are calculated as:

$$
\begin{gathered}
\sigma_{i}^{(1)}=\int_{E_{\min }}^{\infty} d E_{1} N^{(1)}\left(E_{1}\right) \sigma_{A_{p}}\left(E_{1}\right) f_{i}^{(1)}\left(E_{1}\right), \\
\sigma_{i}^{(2)}=\int_{E_{\min }}^{\infty} \int_{E_{\min }}^{\infty} d E_{1} d E_{2} N^{(2)}\left(E_{1}, E_{2}\right) \sigma_{A_{p}}\left(E_{1}\right) \sigma_{A_{p}}\left(E_{2}\right) f_{i}^{(2)}\left(E_{1}, E_{2}\right) .
\end{gathered}
$$

Here we introduce the single and double photon spectral functions which appear after the integration over impact parameters;

$$
\begin{gathered}
N^{(1)}\left(E_{1}\right)=2 \pi \int_{b_{\text {min }}}^{\infty} b d b e^{-m(b)} N\left(E_{1}, b\right), \\
N^{(2)}\left(E_{1}, E_{2}\right)=\pi \int_{b_{\text {min }}}^{\infty} b d b e^{-m(b)} N\left(E_{1}, b\right) N\left(E_{2}, b\right) .
\end{gathered}
$$

In the above expressions $b_{\min }$ is the minimal value of the impact parameter which corresponds to the onset of nuclear interaction. The integration over the photon energy starts from $E_{\min } \approx 7 \mathrm{MeV}$ which corresponds to the threshold of nuclear dissociation in photonuclear reactions. Due to the long-range nature of the electromagnetic forces, numerical calculations of the integrals (11) and (12) should be undertaken with care. A reasonable accuracy can be obtained if one splits the whole region of integration into two intervals: $\left[b_{\min }, b_{c u t}\right]$ and $\left[b_{c u t}, \infty\right)$. The value of $b_{\text {cut }}$ is determined by the condition $e^{-m\left(b_{c u t}\right)} \approx 1$ which allows one to omit the exponential factor in the second interval. After that the first integral in Eq. (11) is calculated numerically, while the second one can be evaluated analytically, leading to a well-known expression 15. The same splitting can be made in the integral (12). Using the asymptotic behaviour at $x \gg 1$ :

$$
K_{1}(\mathrm{x}) \approx \sqrt{\pi / 2 \times} e^{-\mathrm{x}}
$$


one obtains the exponential integral which can be found in mathematical tables. With the described method one can avoid problems with too many nodes in the numerical integration over the wide range of impact parameters.

The values of $f_{i}^{(1)}$ and $f_{i}^{(2)}$ for branching ratios of partial channels of photonuclear dissociation induced by real and virtual photons were calculated within the model of Ref. [6] which takes into account the fast cascade stage of the reaction as well as evaporation, fission and multifragmentation processes for a residual nucleus.

\subsection{Excitation and decay of residual nuclei}

Relative probabilities of the de-excitation processes are determined by the excitation energy $E^{\star}$ of a residual nucleus formed after the completion of the fast cascade stage of the photonuclear reaction. In the following we consider step-bystep all these mechanisms with a special attention to the amount of energy which is transformed on average into the internal excitation of the nucleus.

Depending on the virtual photon energy $E_{\gamma}$ different processes may contribute to the energy deposition. When a nucleus absorbs one or two virtual photons in the GR region, $6 \leq E_{\gamma} \leq 30 \mathrm{MeV}$, their energies are completely transformed into the excitation energy $E^{\star}$. For preactinide nuclei like $\mathrm{Au}$ and $\mathrm{Pb}$, whose fission thresholds are about $30 \mathrm{MeV}$, the de-excitation proceeds mainly through the evaporation of neutrons, since their separation energies are only around 7 $\mathrm{MeV}$. Due to a high Coulomb barrier in heavy nuclei, the proton emission is suppressed in the GR region. A detailed experimental investigation of $(\gamma, n)$, $(\gamma, 2 n),(\gamma, 3 n)$, and $(\gamma, 4 n)$ reactions on ${ }^{197} \mathrm{Au}$ and ${ }^{208} \mathrm{~Pb}$ nuclei in the GR region was performed in Ref. [17. The presence of the reaction $(\gamma, 4 n)$ means that a nucleus has received $E^{\star}$ up to $30 \mathrm{MeV}$. For simulating the particle emission in the evaporation process we used the standard Weisskopf evaporation scheme [7] with the assumption $E^{\star}=E_{\gamma}$.

Starting from $E_{\gamma}=30 \mathrm{MeV}$, where the quasideuteron mechanism becomes important and up to the single pion production threshold at $E_{\gamma}=140 \mathrm{MeV}$, only a part of the photon energy is converted on average into the excitation energy $E^{\star}$ of the compound nucleus. The rest of the photon energy is taken away by the fast nucleons originating from the absorbing pair. The energy deposition is simulated by the IntraNuclear Cascade (INC) model (see Refs. [5, 6] for details). The two-nucleon absorption cross section of a photon on a heavy nucleus, $\sigma_{A}$, is taken from the quasideuteron model of Ref. [18]:

$$
\sigma_{A}^{Q D}=k Z(1-Z / A) \sigma_{d}^{e x c h}
$$

Here $\sigma_{d}^{e x c h}$ is the meson exchange part of the cross section for the deuteron photodisintegration, $\gamma d \rightarrow n p$. This cross section was calculated in Ref. [23]. In Eq. (14) $A$ and $Z$ are the mass and charge numbers of the relevant nucleus 
and $k \approx 11[18$ is an empirical constant. The angular distribution of nucleons in the reaction $\gamma d \rightarrow n p$ was approximated in accordance with empirical fits from Ref. [24]. Although the cross section $\sigma_{d}$ decreases strongly with the photon energy, the two-nucleon absorption mechanism competes noticeably with the single-nucleon photoabsorption channel up to $E_{\gamma} \sim 0.5 \mathrm{GeV}$.

As found in Ref. [18], up to 12 neutrons can be emitted by photo-excited $\mathrm{Pb}$ nucleus. Assuming that the emission comes from the compound nucleus one can conclude that $\left\langle E^{\star}\right\rangle \leq 70-80 \mathrm{MeV}$. The calculations of Ref. [26] for photoabsorption on ${ }^{197} \mathrm{Au}$ and ${ }^{208} \mathrm{~Pb}$ nuclei, performed in the framework of another version of INC model [24], demonstrate that $\left\langle E^{\star}\right\rangle$ does not exceed $80 \mathrm{MeV}$ for $30 \leq E_{\gamma} \leq 140 \mathrm{MeV}$. In this $E_{\gamma}$ region the fissility $P_{f}$ of ${ }^{197} \mathrm{Au}$ and ${ }^{208} \mathrm{~Pb}$ nuclei becomes noticeable $\left(P_{f} \sim 0.01\right.$ and 0.1 , respectively), so one should expect the evaporation-fission competition.

Above the single pion production threshold at $E_{\gamma}=140 \mathrm{MeV}$ the $\Delta$-isobar excitation of individual nucleons dominates. An interesting effect was noticed in Ref. [26]. Near the threshold a slow pion has a small interaction cross section with nucleons and therefore, has a high probability to carry away a large part $\left(\approx m_{\pi}\right)$ of the photon energy. Only at $E_{\gamma} \approx 200 \mathrm{MeV}$ the average value $\left\langle E^{\star}\right\rangle$ starts to increase again. During the fast cascade stage the initial nucleus looses on average 1-2 nucleons and acquires the excitation energy $\left\langle E^{\star}\right\rangle \sim 100 \mathrm{MeV}$, which is sufficient for evaporation of many neutrons or fission. The evaporation may take place before of after the fission. In some cases fission fragments evaporate neutrons as well.

Above the two-pion production threshold at $E_{\gamma} \sim 400 \mathrm{MeV}$ the photonnucleon $(\gamma N)$ interaction becomes more complicated because of many possible final states. Below we use a phenomenological model for exclusive description of the $\gamma N$-interaction developed in Ref. [5]. The model includes both the resonance contribution from the two-body channels, $\gamma N \rightarrow \pi B^{\star}$ and $\gamma N \rightarrow M^{\star} N\left(B^{\star}\right.$ and $M^{\star}$ being baryon and meson resonances), and the non-resonant statistical contribution from the multi-body channels $\gamma N \rightarrow i \pi N(2 \leq i \leq 8)$. Subsequently, the fast hadrons produced in a primary $\gamma N$-interaction initiate a cascade of successive hadron-nucleon collisions inside the nucleus. Finally, when the photon energy reaches the value of several $\mathrm{GeV}$, the multiple pion production becomes the main process. A large number $(\sim 80)$ of many-body subchannels is included in the calculation.

Fig. 11 shows the fraction of $E_{\gamma}$ that on average is transformed into $E^{\star}$ as well as average values of $E^{\star}$ per nucleon of residual nucleus. As it was deduced from the experimental data [25], $E^{\star}=43.4 \pm 5 \mathrm{MeV}$ for photoabsorption on lead at $E_{\gamma}=70 \mathrm{MeV}$. This value agrees well with the prediction of our model (see Fig. 1a). In Fig. 11a one can see the above-mentioned decrease of $\left\langle E^{\star}\right\rangle / E_{\gamma}$ above $E_{\gamma}=140 \mathrm{MeV}$. As seen from Fig. 1, when the virtual photon energy grows from the GR region to several $\mathrm{GeV}$, the nature of the Coulomb excitation changes from the collective nuclear degrees of freedom to the excitation of a single 
nucleon inside the nucleus. In the latter case up to $95 \%$ of the photon energy is released in the form of fast particles leaving the nucleus. Nevertheless, the remaining energy deposited in the residual nucleus is sufficient for evaporating many neutrons. The moments of the neutron multiplicity distribution are shown in Fig. 1, parts c and d. Calculated moments are in a satisfactory agreement with the experimental values for the real photon absorption on $\mathrm{Pb}$ nucleus [25]. Below we use the same model for the case of the virtual photon absorption in electromagnetic interactions of ultrarelativistic heavy ions.

It should be stressed however, that Fig. 1 shows only the average values, while a part of the interactions initiated by "hard" photons will lead to a stronger heating of the nucleus by a multi-pion system. This phenomenon was studied in detail in Ref. [6] were it was shown that the yield of events with high excitation energy, resulting in the multifragment break-up of residual nuclei, might be quite sizable, up to $10 \div 15 \%$ of the total ED cross section. These events can be easily observed with properly designed forward-angle detectors at RHIC and LHC colliders.

To include all the processes described above we have developed a specialized computer code RELDIS aimed at the Monte Carlo simulation of the Relativistic ELectromagnetic DISsociation of nuclei. The simulation begins with generating the single or double-photon absorption process. Then the intranuclear cascade model is used to calculate the fast particle emission and the characteristics of residual nuclei. Finally the de-excitation of thermalized residual nuclei is simulated according to the statistical model [7].

\subsection{Analysis of nucleon emission data}

Let us consider now how the calculations based on Weizsäcker-Williams (WW) method describe the measured 27 cross sections of $1 n, 2 n$ and $3 n$ removal from ${ }^{197} \mathrm{Au}$ nuclei excited electromagnetically. The branching ratios, $f_{i}^{(1)}$ and $f_{i}^{(2)}$, for partial channels of a photonuclear reaction are important ingredients of such calculations. The information concerning these ratios can be obtained from experiments with real photons [17, 20, 25] or from models of photonuclear reactions analogous to the one used in the present paper. We review in brief the advantages and disadvantages of both these approaches.

The calculations [27] within the framework of WW method, which include both isoscalar and isovector quadrupole excitations as well as multiphonon states, describe the one-neutron emission data fairly well. Using the harmonic-oscillator model one can derive a Poisson distribution for the excitation probabilities of multiphonon states. On the contrary, even the theory with these additional modifications is not able to describe the $2 n$ removal cross section. The measured cross section is twice as large as the calculated value, which is determined mainly by the probability of the double-phonon excitation. Further modifications of the WW method, using a "soft-spheres" model and including nuclear-plus-Coulomb 
processes 28], do not remove the discrepancy. The description of the reaction mechanism within the coupled-channel approach [29] with proper account for the nuclear interaction is not able to improve the situation. Only the inclusion of anharmonicities in the internal Hamiltonian and nonlinear terms in the external field, proposed in Ref. [30] and later supported by other authors [31], help to solve the problem. As shown in Ref. [30, 31], a small deviation from the harmonic model may lead to a doubling of the two-phonon excitation probability and to a good description of the data.

The calculations of Refs. [30, 31] are based on the one- and two-neutron emission cross sections measured in experiments with real photons. Thus only a limited number of channels of electromagnetic dissociation was considered. The differences in the total GDR cross section measured by different groups are found to be on the level of $20 \%$ [20]. There exists also an opinion 32] that for the $2 n$ photoemission measurements the quality of the data is even much worse. Different interpretations of the photonuclear data lead to difficulties in determining the contribution of the double-photon absorption. Indeed, the double GDR state may decay via $2 n$ and $3 n$ channels and therefore its admixture depends on the corresponding partial cross sections.

In the present paper we analyse the ED data 27] within the model of photonuclear reactions introduced above. We do not use experimental data as an input for calculations, except of the information concerning the non-statistical contribution in the $1 n$ emission channel. It should be reminded, that the statistical decay of the compound nucleus is not the only mechanism responsible for the decay of the collective GDR state. This state can also decay directly into a free nucleon and a hole in the residual nucleus, due to the two-body residual interaction in the final state (see Refs. [17, 33] for details). Such a direct neutron emission leaves the residual nucleus in a low-lying energy state where only a subsequent emission of photons is possible. This makes the emission of a second neutron impossible, leading to a suppression of the $(\gamma, 2 n)$ channel in comparison with the pure statistical decay. We include this additional non-statistical contribution in the $(\gamma, n)$ channel on the basis of the experimental information concerning this decay mode. The total fraction of the non-statistical contribution was evaluated to about $35 \%$ and $20 \%$ for $\mathrm{Au}$ and $\mathrm{Pb}$ nuclei, respectively [17]. We use these values in our simulations. The emission angles of non-statistical fast photoneutrons were generated according to the angular distributions measured in Ref. [34.

In our model of photonuclear reactions, implemented in the RELDIS code, we consider all possible decay modes, including binary fission and multifragmentation. It gives the yields and spectra of different particles in the ED reactions induced by real and virtual photons. Before discussing the predictions in the remaining part of the paper, let us consider first how the model describes the existing data on a few neutron removal from Au nuclei [27].

The results for the few-nucleon emission are summarized in Table 11. One can 
see that the model describes the $3 n$ emission quite well but the agreement with the measured one- and two-neutron emission cross sections is not as good. At the same time the total neutron $(n-3 n)$ emission cross section agrees with the experimental value. Due to the statistical nature of our model one can expect that it is not so accurate for the particle emission from collective states like GDR, but works better for the multiple emission from the compound nucleus. This tendency is clearly seen in Table 1. The higher is the energy of colliding ions the greater is the number of emitted particles. Therefore, the quality of description of the low energy data 27 may be considered as a low limit for the accuracy of the model which is expected to work better with increasing energy. It should be noticed that a simple Poisson distributions for multiple excitations was adopted in our calculations. And even within this assumption the agreement with data 27 is acceptable, in contrast with observations of Refs. 27, 28]. This discrepancy is due to slightly different values of the partial photonuclear cross sections ( $2 n$ and $3 n$ ) used in our calculations as compared with the papers [27, 28.

The importance of the double-photon absorption mechanism is illustrated in Tab. 1 too. Although the total cross section of double-photon absorption, $\sigma^{(2)}=226 \mathrm{mb}$, is small compared to the single photon one, $\sigma^{(1)}=3546 \mathrm{mb}$, this contribution is very significant for the rare channels like $(\gamma, 4 n),(\gamma, 5 n)$, $(\gamma, 6 n)$ and, especially, for $(\gamma, p 3 n),(\gamma, p 4 n)$ and $(\gamma, p 5 n)$ channels. These listed channels are extremely sensitive to the presence of the double photon absorption mechanism.

\section{Predictions for ED at $\mathrm{RHIC}$ and $\mathrm{LHC}$}

\subsection{Total and partial ED cross sections}

Let us consider now the model predictions for the electromagnetic dissociation of ultrarelativistic $\mathrm{Pb}$ and $\mathrm{Au}$ nuclei on fixed targets at SPS and in colliding beams at RHIC and LHC. The total ED cross section, $\sigma_{E D}=\sigma^{(1)}+\sigma^{(2)}$, is obtained from Eqs. (9) and (10) by taking $f_{i}^{(1)}=1$ and $f_{i}^{(2)}=1$. The $\sigma_{E D}$ values for most interesting reactions are presented in Tab. 2 .

The inclusive (multiplicity weighted) cross sections for emission of nucleons, nuclear fragments and pions in the electromagnetic dissociation of one of the colliding $\mathrm{Au}$ nuclei are shown in Fig. 2 as functions of the beam energy. In addition to Fig. 2, where the calculations are presented only for $\mathrm{Au}+\mathrm{Au}$ reaction, in Tab. 3 the inclusive cross sections are given for the three reactions listed in Tab. 2. One can see that the partial cross sections for neutron emission are especially large. This is due to the high average neutron multiplicities, 4.1, 7.2 and 8.8, at SPS, RHIC and LHC energies, respectively.

The relative contributions to the inclusive cross sections from the double photon absorption, $\sigma_{i}^{(2)} /\left(\sigma_{i}^{(1)}+\sigma_{i}^{(2)}\right)$, are listed for the same reactions in Tab. 6 . By 
inspecting Tab. 国 one can conclude that about 10\%, 5\% and 2\% of particles, on average, are produced in the second order processes at SPS, RHIC and LHC energies, respectively. These values should be compared with the corresponding contributions to the total ED cross sections (Tab. 2), $\sigma^{(2)} / \sigma_{E D}$, which are, $3.5 \%$, $2.3 \%$ and $1.4 \%$, respectively. Thus, the naive expectation, that in the double photon absorption the multiplicities of particles should be exactly twice as large as in the single photon absorption, is not valid. Indeed, according to this expectation the partial fractions should be twice as large as the fractions of $\sigma^{(2)}$ in the total cross section, i.e. $7 \%, 4.6 \%$ and $2.8 \%$. This rule is violated for the intermediate mass and fission fragments. Nevertheless the general tendency is obvious: high order corrections become less and less important with increasing energy. This is true not only for the total ED cross section, as it was recently noticed in Ref. [35], but also for the partial cross sections for nucleon and fragment emission and pion production.

\subsection{Neutron multiplicity distributions}

As stressed above, neutrons are the most abundant particles produced in the ED process. In addition, there are many mechanisms of neutron emission that makes the calculation of their characteristics especially difficult. During the fast stage of the photonuclear reaction some intranuclear nucleons may escape from the nucleus via a direct knock-out either by the incoming photon or by a secondary particle in the course of the intranuclear cascade. In addition, as it was mentioned before, many neutrons may be evaporated from an excited residual nucleus.

The neutron multiplicity distributions predicted by the RELDIS code are presented in Fig. 0 for the three reactions discussed above. One can see that these distributions have a non-trivial structure. They are strongly peaked at the $1 n$ emission channel associated with the GR decay. On the other hand, there is a long tail of multiple neutron emission associated with the knock-out and evaporation processes. One can see, for example, that the probability to emit more than 20 neutrons is quite noticeable $(\approx 5 \%$ at RHIC). The mean neutron multiplicities for the three reactions considered are 4.2, 7.2 and 8.8 at SPS, RHIC and LHC energies, respectively. These results might be important for designing zero-degree calorimeters at RHIC and LHC.

\subsection{Spectra of nucleons, pions and nuclear fragments}

Now let us discuss the differential distributions in rapidity $y_{l a b}$ and transverse momentum $P_{t}$ for nucleons, nuclear fragments and pions.

The Lorentz-invariant inclusive differential cross sections, $d \sigma / d y_{l a b}$ and $d \sigma / P_{t} d P_{t}$, for neutrons are shown in Fig. 4 . Obviously, the dominant part of neutrons is concentrated near the beam rapidity (see upper panel). However, the cross section of neutron emission is quite large $(\sim 0.1-1 \mathrm{~b})$ even 2-3 units away from the beam 
rapidity. In our model these neutrons are produced due to reactions $\gamma N \rightarrow i \pi n$ $(1 \leq i \leq 8)$ initiated by a high-energy virtual photon. The recoil neutron in these reactions has a chance to keep a great part of the photon energy.

The neutron $P_{t}$ distributions (lower panel) reveal clearly the presence of two components associated with fast and slow stages of the reaction. The evaporation neutrons are concentrated mainly at $P_{t} \leq 150 \mathrm{MeV}$, while the high momentum tail of the $P_{t}$-distribution is formed by early emitted knock-out neutrons.

Fig. 5 shows the invariant differential cross sections, $d \sigma / d y_{l a b}$ and $d \sigma / P_{t} d P_{t}$ for protons. Although the proton rapidity distributions are similar to those for neutrons, their transverse momentum spectra reveal a difference. Namely, the evaporation component in the proton spectra is suppressed by the Coulomb barrier. Instead of two components, a broad peak is seen at $P_{t} \sim 150-250 \mathrm{MeV} / \mathrm{c}$. Its origin can be understood as follows. A virtual photon from the region of quasideuteron absorption, $E_{\gamma} \sim 30-100 \mathrm{MeV}$, produces a pair of nucleons with approximately equal momenta of 200-300 MeV/d . They are emitted predominantly at angles $80^{\circ}-85^{\circ}$ with respect to the collision axis in the nuclear rest frame and therefore the corresponding $P_{t}$ is approximately $150-250 \mathrm{MeV} / \mathrm{c}$. This prediction can be checked by measuring the $P_{t}$ distributions of protons produced in the ED reactions. Up to now mainly the collective excitations of nuclei in the GR region were studied in these reactions through the neutron emission [27] or fission [36] channels. There were no special attempts to see how virtual photons interact directly with individual nucleons.

The transverse momentum distributions of intermediate-mass $(3 \leq Z \leq 30)$ and fission $(30<Z \leq 50)$ fragments are shown in Fig. 6. They are remarkably flat. All these fragments are produced at late stages of the reaction and their characteristic momenta, in the nuclear rest frame, are of about $100 \mathrm{MeV} / \mathrm{c}$ per nucleon. Their rapidity distributions are not shown because they are extremely narrow and centered around the beam rapidities. As shown in Ref. [6], shell structure effects do not play a role at these high excitation energies and, therefore, fission fragments have a symmetric charge distribution. The distribution of intermediate-mass fragments is close to a power law.

\subsection{Background for $\gamma \gamma$ processes}

In this section we demonstrate that pions produced in the $\gamma A$ process may cause problems in studying $\gamma \gamma$ fusion reactions. The predicted distributions of positive and negative pions from $\gamma A$ processes are given in Figs. 7 and 8, respectively. Both $\pi^{+}$and $\pi^{-}$have very wide rapidity distributions which extend toward midrapidity up to $\left|y_{l a b}\right|<2$ for LHC and up to $y_{l a b} \sim 0$ for RHIC. One can also notice that some pions have rapidities which exceed the beam rapidity $\left(y_{R H I C}=5.4\right.$, $\left.y_{L H C}=8.3\right)$. This is a peculiarity of the multiple pion photoproduction at high

\footnotetext{
${ }^{1}$ Fermi motion is neglected in this estimation.
} 
energies when a large phase space is available for pions. Some pions are emitted backward with respect to the virtual photon momentum.

The pions produced in $\gamma A$ interactions close to the mid-rapidity region may create complications for the detection of particles produced from $\gamma \gamma$-fusion process [8, 9, 10]. This can be seen from a simple estimate. As predicted in Ref. [10], the cross section to produce a $D_{s}^{+}$meson at LHC is $0.144 \mathrm{mb}$ and $d \sigma / d y_{\text {lab }}$ is peaked at $y_{l a b}=0$ so that almost all these mesons will have rapidities within the interval $\left|y_{l a b}\right|<2$. The probabilities of $D_{s}^{+}$decays into $\eta \pi^{+}, \omega \pi^{+}$or $\rho^{0} \pi^{+}$channels are expected at the level of $1-5 \%$, [37]. Then the cross section to produce a $\pi^{+}$from the $D_{s}^{+}$decay turns out to be below $0.007 \mathrm{mb}$. On the other hand, for $\pi^{+}$from the $\gamma A$ process we predict $d \sigma / d y_{l a b} \sim 10 \mathrm{mb}$ at $\left|y_{l a b}\right|=2$ (see Fig. (7). These estimations show that it will be very difficult to separate the products of the $D_{s}^{+}$decay from the background of the $\gamma A$ process. This does not necessarily mean that the detection of $D_{s}^{+}$is impossible since different kinematical cuts may be applied to suppress the $\gamma A$ background, as well as other decay modes may be used for the $D_{s}^{+}$detection. The considered example rather demonstrates the importance of the proper evaluation of the $\gamma A$ background in planning experiments on $\gamma \gamma$ fusion at future colliders.

\section{Conclusions}

In this paper we have further developed the model of electromagnetic dissociation of nuclei first introduced in Ref. [6]. The computer code RELDIS is developed to calculate a wide range of observable characteristics associated with the ED reactions. This code includes the dissociation channels with nucleons, pions, fission fragments and intermediate mass fragments from nuclear multifragmentation. The model calculations are in a good agreement with experimental data obtained for the dissociation of $1 \mathrm{GeV} /$ nucleon ${ }^{197} \mathrm{Au}$ ions. This gives us the basis to put forward predictions for the electromagnetic dissociation of ultrarelativistic heavy ions at RHIC and LHC energies. One should bear in mind that the multiplicities of particle produced by high-energy virtual photons are rather small as compared with those in central nuclear collisions. But the reaction rates are large. Besides the fundamental interest, the ED process may have interesting applications. As proposed in Ref. [38], the neutron emission in the ED process may be used for luminosity monitoring via zero degree calorimeters. Detailed calculations presented in this paper make it possible to eliminate some unnecessary simplifications in evaluating the neutron transverse momentum distributions which were adopted in Ref. [38]. Although the advantages of detecting mutual one-neutron dissociation events are prominent [39], one should carefully estimate the contribution of more deep dissociation processes, as it has been made in the present paper. The electromagnetic fission of $\mathrm{Au}$ and $\mathrm{Pb}$ nuclei will be also noticeable at RHIC and LHC. An alternative approach to luminosity monitoring 
would be to measure the yields of fission fragments or protons, which can be easily separated from the beam nuclei by their different Z/A-ratio. Using presented above $P_{t}$ distributions of fission and intermediate mass fragments one can estimate the response of forward detectors to such fragments. The calculated differential distributions of pions should be taken into consideration in planning experiments on $\gamma \gamma$ fusion at CERN [10].

One can use the predictions of the present model in order to disentangle different mechanisms of the heavy-ion dissociation, not only though the measurement of the Z-dependence of the total cross section, as it was made in Ref. [41, but also through the registration of some specific exclusive channels (multiple neutron emission, for example). Such measurements are needed, in particular, for understanding the origin of the correction term in the dissociation cross section found in Ref. [41].

We are grateful to J.J.Gaardhøje, A.B.Kurepin, M.V.Mebel and L.M.Satarov for useful discussions. I.N.M., A.S.B. and I.A.P. thank the Niels Bohr Institute for the warm hospitality and financial support. I.A.P. thanks INTAS for the Young Scientists Fellowship 98-86. The work was supported partially by the Danish Natural Science Research Council and Universities of Russia Basic Research Fund, grant 5347 .

\section{References}

[1] I.N. Mishustin, L.M. Satarov, H. Stocker, W. Greiner, Phys. Rev. C 52, 3315 (1995).

[2] I.N. Mishustin, L.M. Satarov, H. Stocker, W. Greiner, Phys. Rev. C 57, 2552 (1998).

[3] A. Winther and K. Alder, Nucl. Phys. A319, 518 (1979).

[4] C.A. Bertulani and G. Baur, Phys. Rep. 163, 299 (1988).

[5] A.S.Iljinov, I.A.Pshenichnov, N.Bianchi, E.De Sanctis, V.Muccifora, M.Mirazita, P.Rossi, Nucl. Phys. A616, 575 (1997).

[6] I.A. Pshenichnov, I.N. Mishustin, J.P. Bondorf, A.S. Botvina, A.S. Iljinov, Phys. Rev. C 57, 1920 (1998).

[7] J.P.Bondorf, A.S.Botvina, A.S.Iljinov, I.N.Mishustin and K.Sneppen, Phys. Rep. 257, 133 (1995).

[8] M.Greiner, M.Vidovic and G.Soff, Phys. Rev. C 47, 2288 (1993).

[9] N. Baron and G. Baur, Phys. Rev. C 49, 1127 (1994). 
[10] M. Vidovic, M. Greiner and G. Soff, J. Phys. G 21, 545 (1995).

[11] K. Hencken, D. Trautmann, G. Baur, Z. Phys. C 68, 473 (1995).

[12] ALICE - Technical Proposal for A Large Ion Collider Experiment at the CERN LHC, CERN/LHCC/95-71, December 1995.

[13] R.Engel, M.A.Braun, C.Pajares and J.Ranft, Z. Phys. C 74, 687 (1997).

[14] R.Engel, Z. Phys. C 66203 (1995); R.Engel and J.Ranft, Phys. Rev. D 54, 4244 (1996).

[15] J.D.Jackson, Classical electrodynamics, 2nd ed. (Wiley, New York, 1975).

[16] W.J.Llope and P.Braun-Munzinger, Phys. Rev. C 41, 2644 (1990).

[17] A.Veyssière, H.Beil, R.Bergère, P.Carlos and A.Leprêtre, Nucl. Phys. A159, 561 (1970).

[18] A.Leprêtre, H.Beil, R.Bergère, P.Carlos, J.Fagot, A.De Miniac and A.Veyssière, Nucl. Phys. A367, 237 (1981).

[19] R.R.Harvey, J.T.Caldwell, R.L.Bramblett and S.C.Fultz, Phys. Rev. 136, 126 (1964).

[20] B.L.Berman, R.E.Pywell, S.S.Dietrich, M.N.Thompson, K.G.McNeill, J.W.Jury, Phys. Rev. C 36, 1286 (1987).

[21] M.Mirazita, H.Avakian, N.Bianchi, A.Deppman, E.De Sanctis, V.Gyurjyan, V.Muccifora, E.Polli, P.Rossi, R.Burgwinkel, J.Hannappel, F.Klein, D.Menze, W.Schwille, F.Wehnes, Phys. Lett. B 407, 225 (1997).

[22] B.L.Berman and S.C.Fultz, Rev. Mod. Phys. 47, 713 (1975).

[23] J.M.Laget, Nucl.Phys. A312, 256 (1978).

[24] V.S.Barashenkov, F.G.Gereghi, A.S.Iljinov, G.G.Jonsson and V.D.Toneev, Nucl. Phys. A231, 462 (1974).

[25] A.Leprêtre, H.Beil, R.Bergère, P.Carlos, J.Fagot, A.De Miniac and A.Veyssière, Nucl. Phys. A390, 221 (1982).

[26] C.Guaraldo, V.Lucherini, E.De Sanctis. A.S.Iljinov, M.V.Mebel, S.Lo Nigro, Nuovo Cim. A103, 607 (1990).

[27] T.Aumann, J.V.Kratz, E.Stiel, K.Summerer, W.Bruchle, M.Shadel, G.Wirth, M.Fauerbach, J.C.Hill, Phys. Rev. C 47, 1728 (1993).

[28] T.Aumann, C.A.Bertulani and K.Summerer, Phys. Rev. C 51, 416 (1993). 
[29] C.A.Bertulani, L.F.Canto, M.S.Hussein, A.F.R.de Toledo Piza, Phys. Rev. C 53, 334 (1996).

[30] C.Volpe, F.Catara, Ph.Chomaz. M.V.Andres and E.G.Lanza, Nucl. Phys. A589, 521 (1995).

[31] P.F.Bortignon and C.H.Dasso, Phys. Rev. C 56, 574 (1997).

[32] E. Wolynec, A.R.V. Martinez, P. Gouffon, Y. Miyao, V.A. Serrao, M.N. Martins, Phys. Rev. C 29, 1137 (1984).

[33] R.E.Van de Vyver, E.Van Camp, D.R.Ryckbosch, E.Kerkhove, P.Van Otten, P.Berkvens and H.Ferdinande, In: Proc. of 5th Seminar on Electromagnetic Interaction of Nuclei at Low and Medium Energies (Moscow, 1981), p.135.

[34] F.Tagliabue and J.Goldemberg, Nucl. Phys. 23, 144 (1961).

[35] J.Norbury and M.L.Waldsmith, Phys. Rev. C 57, 1525 (1998).

[36] Th.Rubehn, W.F.Muller, R.Bassini, M.Begemann-Blaich, T.Blaich, A.Ferrero, C.Gross, G.Imme, I.Iori, G.J.Kunde, W.D.Kunze, V.Lindenstruth, U.Lynen, T.Mohlenkamp, L.G.Moretto, B.Oscker, J.Pochodzalla, G.Raciti, S.Reito, H.Sann, A.Schuttauf, W.Seidel, V.Serfling, W.Trautmann, A.Trzcinski, G.Verde, A.Worner, E.Zude, B.Zweiglinsky, Z. Phys. A353, 197 (1995).

[37] Particle Data Group (R.M.Barnett et al.), Phys. Rev. D 54, 1 (1996).

[38] A.J.Baltz and S.N.White, RHIC/DET-20, Preprint BNL-63127

[39] A.J.Baltz, C.Chasman, S.N.White, Nucl. Instrum. Meth. A417, 1 (1998).

[40] G.Baur, K.Hencken, D.Trautmann, S.Sadovsky and Yu.Kharlov, CMS NOTE 1998/009, January 26, 1998

[41] S.Datz, J.R.Beene, P.Grafström, H.Knudsen. H.F.Krause, R.H.Schuch and C.R.Vane, Phys. Rev. Lett. 79, 3355 (1997). 
Table 1: Calculated and measured [27] cross sections for nucleon emission in the reactions ${ }^{197} \mathrm{Au}\left({ }^{197} \mathrm{Au}, x n\right){ }^{197-x} \mathrm{Au}$ and ${ }^{197} \mathrm{Au}\left({ }^{197} \mathrm{Au}, p x n\right){ }^{196-x} \mathrm{Pt}$ at $1 \mathrm{~A} \mathrm{GeV}$.

\begin{tabular}{|c|c|c|c|c|}
\hline \hline \multirow{2}{*}{ Channel } & \multicolumn{4}{|c|}{ Cross section (mb) } \\
\cline { 2 - 5 } & \multicolumn{3}{|c|}{ Model } & Experiment \\
& \multirow{3}{*}{$\sigma_{i}^{(1)}$} & $\sigma_{i}^{(2)}$ & $\sigma_{i}$ & $\sigma_{i}$ \\
\cline { 2 - 5 } & & & & \\
\hline \hline$n$ & 2432 & 74 & 2506 & $3077 \pm 200$ \\
$2 n$ & 926 & 13 & 939 & $643 \pm 105$ \\
$3 n$ & 129 & 77 & 206 & $171 \pm 26$ \\
\hline Total & & & & \\
$n-3 n$ & 3487 & 164 & 3651 & $3891 \pm 227$ \\
\hline \hline $4 n$ & 40 & 43 & 83 & - \\
$5 n$ & 7.7 & 11.6 & 19.3 & - \\
$6 n$ & 2.2 & 4.4 & 6.6 & - \\
\hline Total & & & & \\
$4 n-6 n$ & 49.9 & 59 & 108.9 & - \\
\hline \hline$p$ & 1.7 & 0 & 1.7 & - \\
$p n$ & 3.2 & 0 & 3.2 & - \\
$p 2 n$ & 2.5 & 0.6 & 3.1 & - \\
\hline Total & & & & \\
$p-p 2 n$ & 7.4 & 0.6 & 8 & - \\
\hline \hline$p 3 n$ & 0.8 & 0.7 & 1.5 & - \\
$p 4 n$ & 0.3 & 0.5 & 0.8 & - \\
$p 5 n$ & 0.1 & 0.2 & 0.3 & - \\
\hline Total & & & & \\
$p 3 n-p 5 n$ & 1.2 & 1.4 & 2.6 & - \\
\hline \hline & & &
\end{tabular}


Table 2: Total cross sections (barn) of electromagnetic dissociation, $\sigma_{E D}$, and contributions from the single, $\sigma^{(1)}$, and double, $\sigma^{(2)}$, photon absorption. The values are given for the present and future heavy-ion beams of $\mathrm{Pb}$ and $\mathrm{Au}$ nuclei.

\begin{tabular}{|c|c|c|c|}
\hline \hline Reaction & $\sigma^{(1)}$ & $\sigma^{(2)}$ & $\sigma_{E D}$ \\
\hline \hline $\begin{array}{c}158 \mathrm{~A} \mathrm{GeV} \\
208 \mathrm{~Pb} \mathrm{on}{ }^{208} \mathrm{~Pb}\end{array}$ & 41.2 & 1.5 & 42.7 \\
\hline $\begin{array}{c}100 \mathrm{~A}+100 \mathrm{~A} \mathrm{GeV} \\
197 \mathrm{Au} \text { on }{ }^{197} \mathrm{Au}\end{array}$ & 93.8 & 2.2 & 96 \\
\hline $\begin{array}{c}2.75 \mathrm{~A}+2.75 \mathrm{~A} \mathrm{TeV} \\
208 \mathrm{~Pb} \text { on }{ }^{208} \mathrm{~Pb}\end{array}$ & 208.7 & 3 & 211.7 \\
\hline \hline
\end{tabular}

Table 3: Inclusive cross sections (barn) of emission of nucleons, nuclear fragments and pions in the electromagnetic dissociation of $\mathrm{Pb}$ and $\mathrm{Au}$ nuclei calculated for the present and future RHI beams.

\begin{tabular}{|c|c|c|c|c|c|c|c|}
\hline \hline \multirow{2}{*}{ Reaction } & \multicolumn{9}{|c|}{$\sigma_{i}^{(1)}+\sigma_{i}^{(2)}$} \\
\cline { 2 - 8 } & $p$ & $n$ & $3 \leq Z \leq 30$ & $30<Z \leq 50$ & $\pi^{+}$ & $\pi^{-}$ & $\pi^{0}$ \\
\hline \hline $\begin{array}{c}158 \mathrm{~A} \mathrm{GeV} \\
208 \\
\mathrm{~Pb} \text { on }{ }^{208} \mathrm{~Pb}\end{array}$ & 15.9 & 175 & 0.09 & 0.5 & 1.37 & 2.28 & 2.7 \\
\hline $\begin{array}{c}100 \mathrm{~A}+100 \mathrm{~A} \mathrm{GeV} \\
197 \mathrm{Au} \text { on }{ }^{197} \mathrm{Au}\end{array}$ & 104.7 & 686.7 & 6.28 & 4.3 & 24 & 30.2 & 33.1 \\
\hline $\begin{array}{c}2.75 \mathrm{~A}+2.75 \mathrm{~A} \mathrm{TeV} \\
208 \mathrm{~Pb} \text { on }{ }^{208} \mathrm{~Pb}\end{array}$ & 302.4 & 1853 & 22.3 & 11.9 & 90.4 & 111.6 & 121.4 \\
\hline \hline
\end{tabular}

Table 4: Electromagnetic dissociation of $\mathrm{Pb}$ and $\mathrm{Au}$ nuclei. Contributions (\%) due to the double photon absorption to the inclusive cross sections of emission of nucleons, nuclear fragments and pions.

\begin{tabular}{|c|c|c|c|c|c|c|c|}
\hline \hline \multirow{2}{*}{ Reaction } & \multicolumn{7}{|c|}{$\sigma_{i}^{(2)} /\left(\sigma_{i}^{(1)}+\sigma_{i}^{(2)}\right)$} \\
\cline { 2 - 9 } & $p$ & $n$ & $3 \leq Z \leq 30$ & $30<Z \leq 50$ & $\pi^{+}$ & $\pi^{-}$ & $\pi^{0}$ \\
\hline \hline $\begin{array}{c}158 \mathrm{~A} \mathrm{GeV} \\
208 \mathrm{~Pb} \text { on }{ }^{208} \mathrm{~Pb}\end{array}$ & 8.1 & 6.7 & 14. & 10.5 & 11.6 & 11.1 & 10.1 \\
\hline $\begin{array}{c}100 \mathrm{~A}+100 \mathrm{~A} \mathrm{GeV} \\
197 \mathrm{Au} \text { on }{ }^{197} \mathrm{Au}\end{array}$ & 3.7 & 3.3 & 4.2 & 4. & 5.6 & 5.3 & 5.06 \\
\hline $\begin{array}{c}2.75 \mathrm{~A}+2.75 \mathrm{~A} \mathrm{TeV} \\
208 \mathrm{~Pb} \text { on }{ }^{208} \mathrm{~Pb}\end{array}$ & 1.8 & 1.8 & 1.4 & 1.8 & 2.4 & 2.4 & 2.3 \\
\hline \hline
\end{tabular}




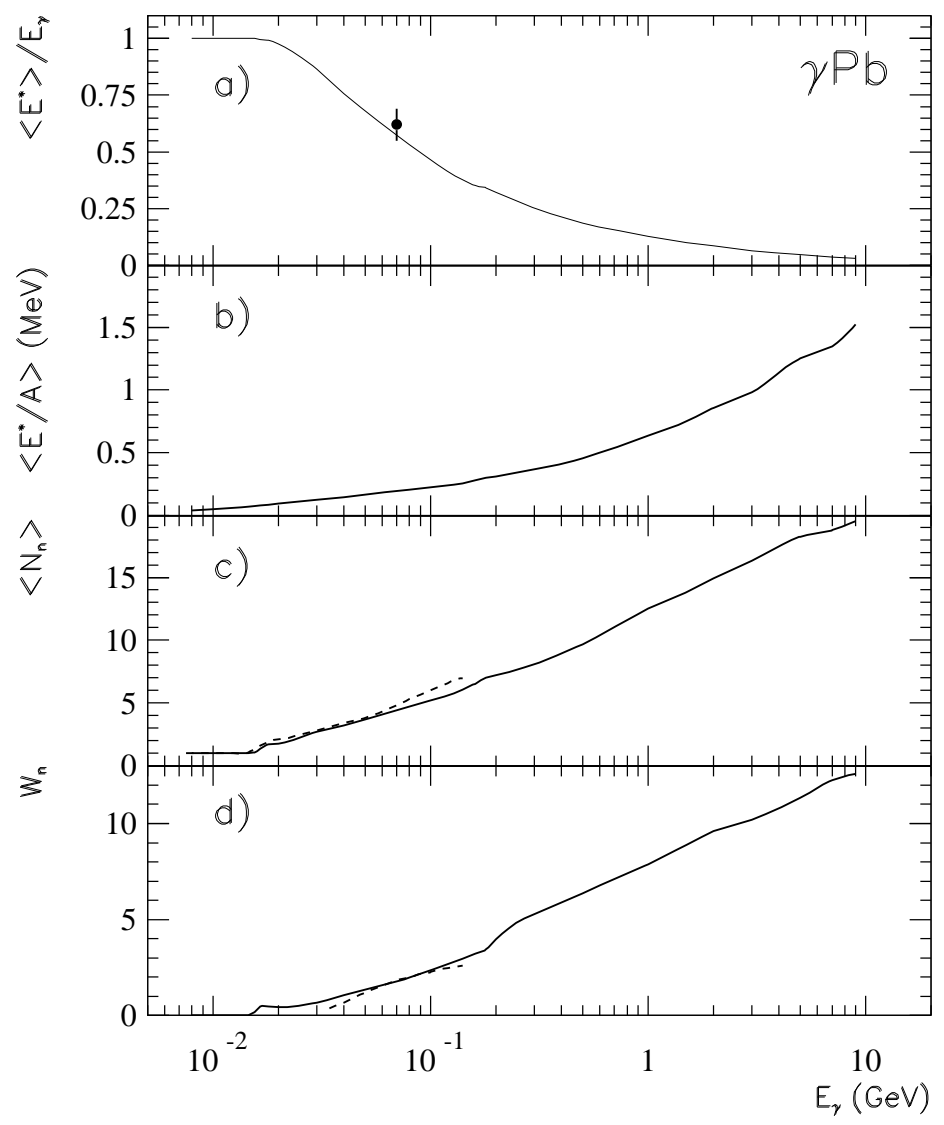

Figure 1: a) Ratio of average excitation energy of residual nucleus $E^{\star}$ to input photon energy $E_{\gamma}$, b) absolute values of $E^{\star}$ per nucleon of residual nuclei, c) average photoneutron multiplicities $\left\langle N_{n}\right\rangle$ and d) width of photoneutron multiplicity $W_{n}=\sqrt{\left\langle N_{n}^{2}\right\rangle-\left\langle N_{n}\right\rangle^{2}}$ as functions of $E_{\gamma}$ in photoabsorption on lead nucleus. Solid lines show the RELDIS calculations, dashed lines and point represent the experimental data 25]. 


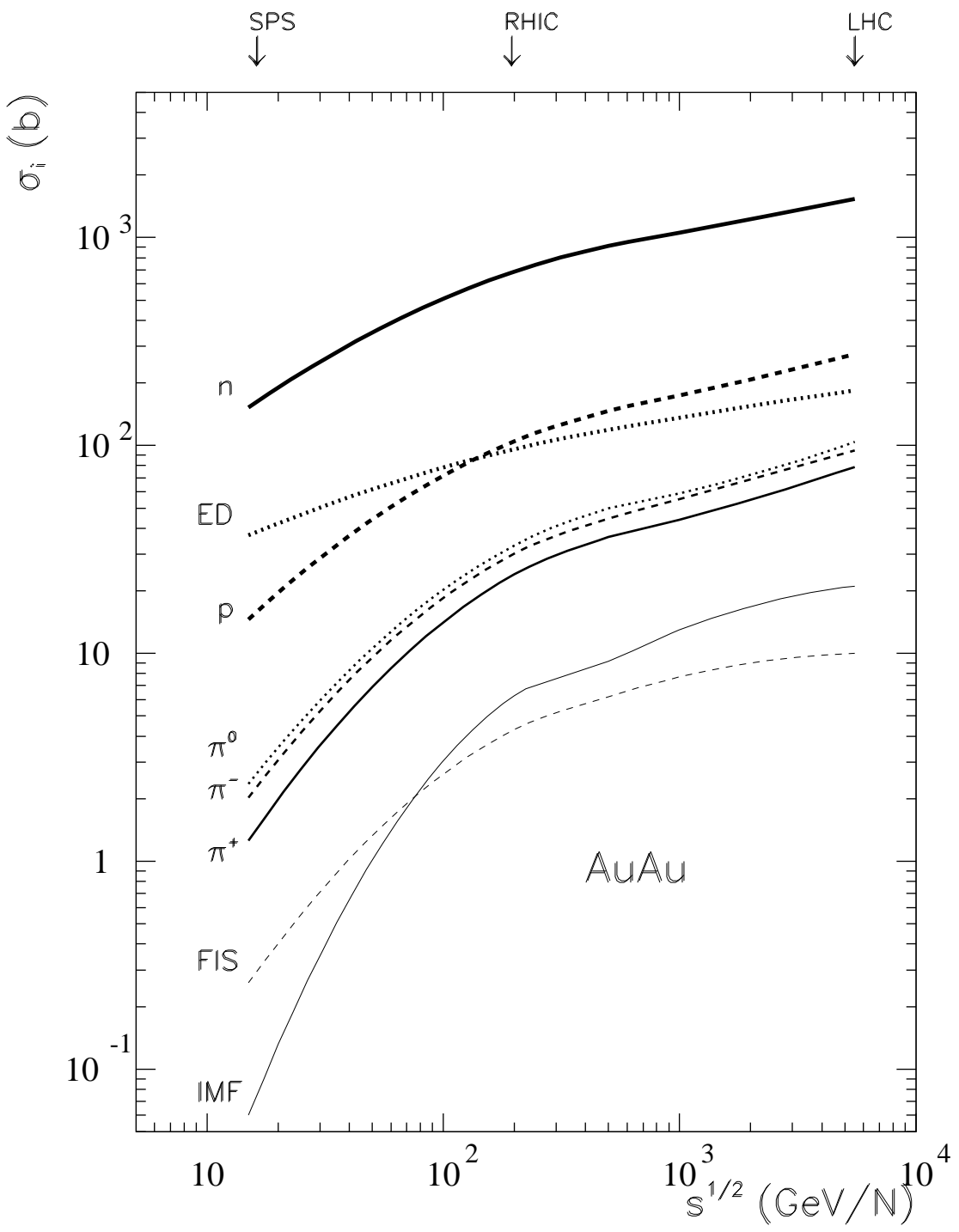

Figure 2: RELDIS predictions for inclusive cross sections of emission of nucleons, nuclear fragments and pions in the electromagnetic dissociation of one of the colliding $\mathrm{Au}$ nuclei. Cross sections for intermediate mass $(3 \leq Z \leq 30)$ and fission $(30<Z \leq 50)$ fragments are labelled as "IMF" and "FIS", respectively. Thick dotted line shows the total electromagnetic dissociation cross section. $\sqrt{s}$ values for SPS, RHIC and LHC are shown by arrows. 


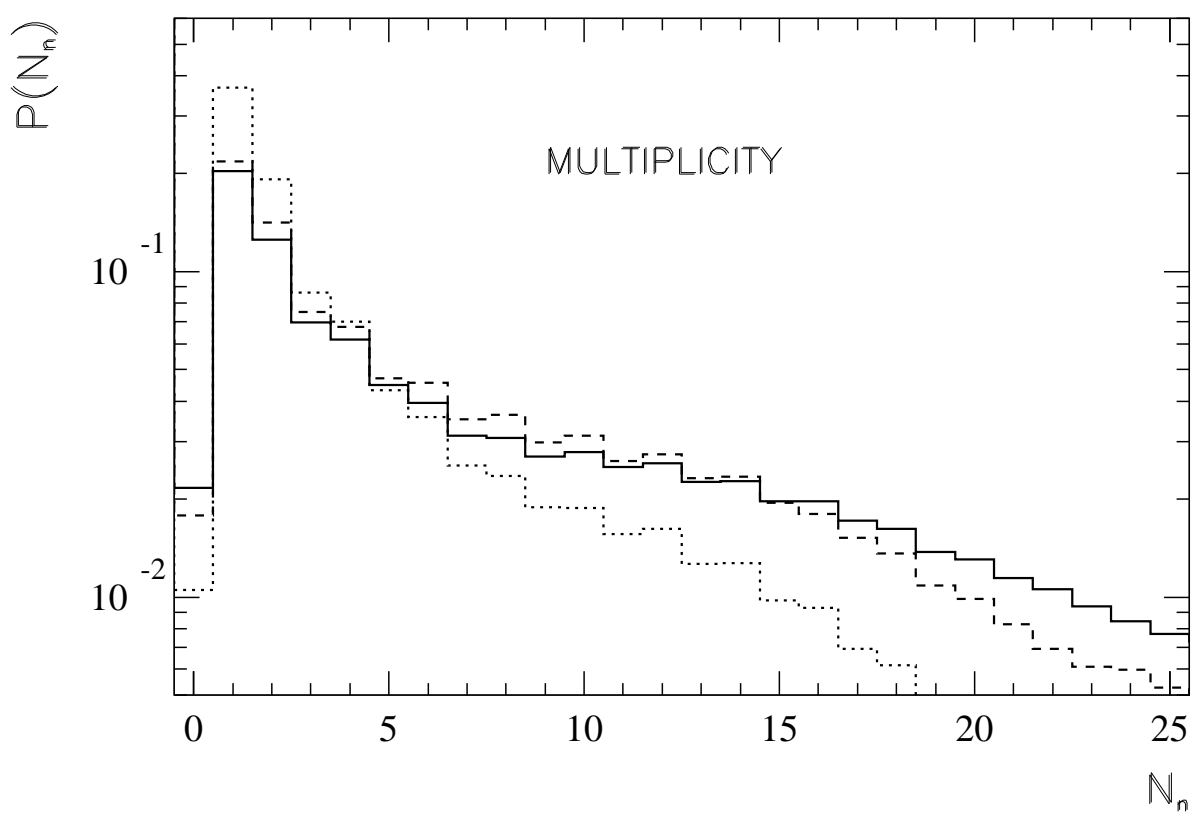

Figure 3: RELDIS predictions for multiplicity distributions of neutrons in the electromagnetic dissociation of $\mathrm{Pb}$ nuclei at LHC and SPS energies (solid and dotted lines, respectively) and Au nuclei at RHIC energies (dashed lines). 

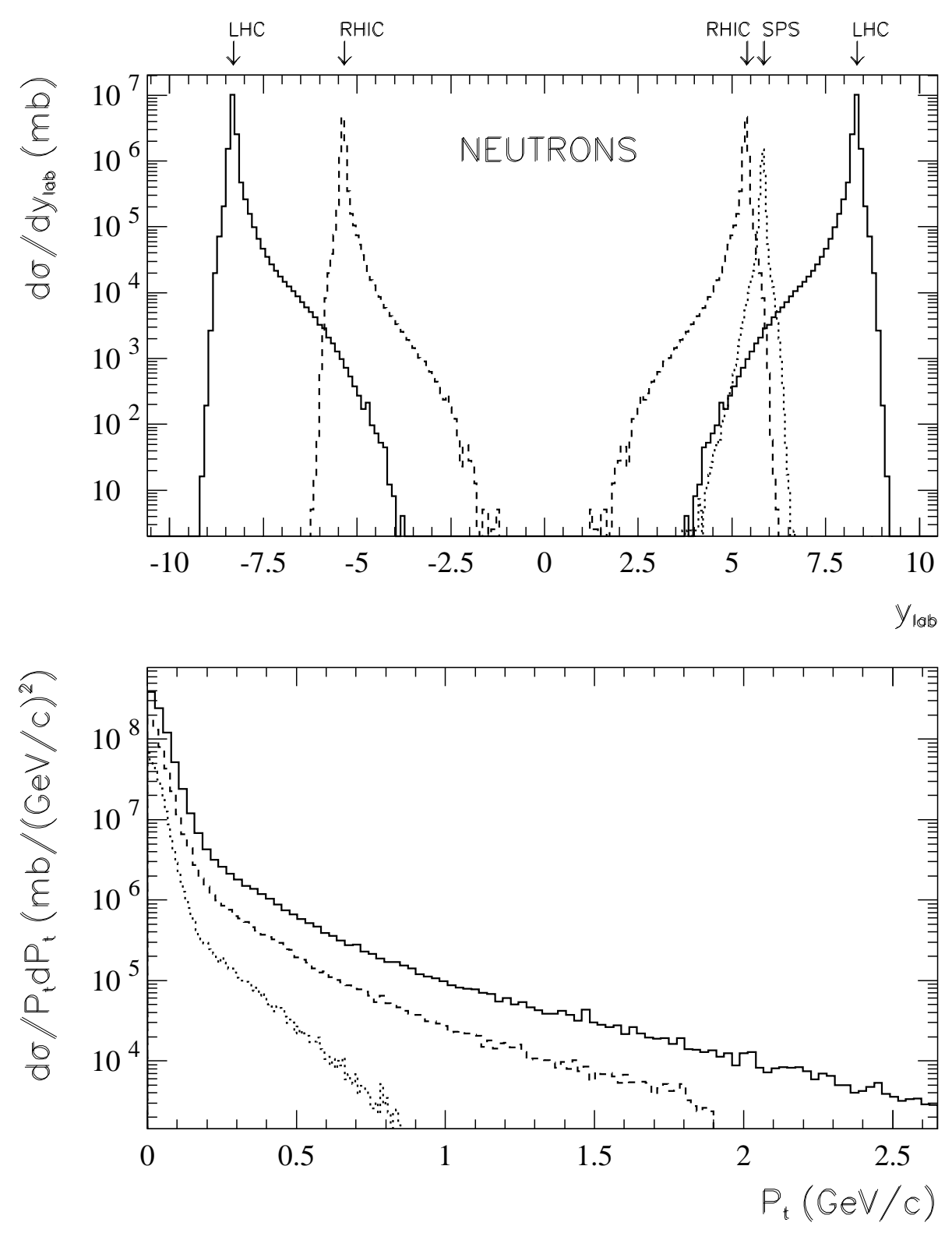

Figure 4: RELDIS predictions for inclusive rapidity (top panel) and transverse momentum (bottom panel) distributions of neutrons emitted in the electromagnetic dissociation of $\mathrm{Pb}$ nuclei at LHC and SPS energies (solid and dotted lines, respectively) and Au nuclei at RHIC energies (dashed lines). Rapidity distributions for colliders were reflected respect to the value $y_{l a b}=0$. Arrows show rapidity of corresponding beams. 

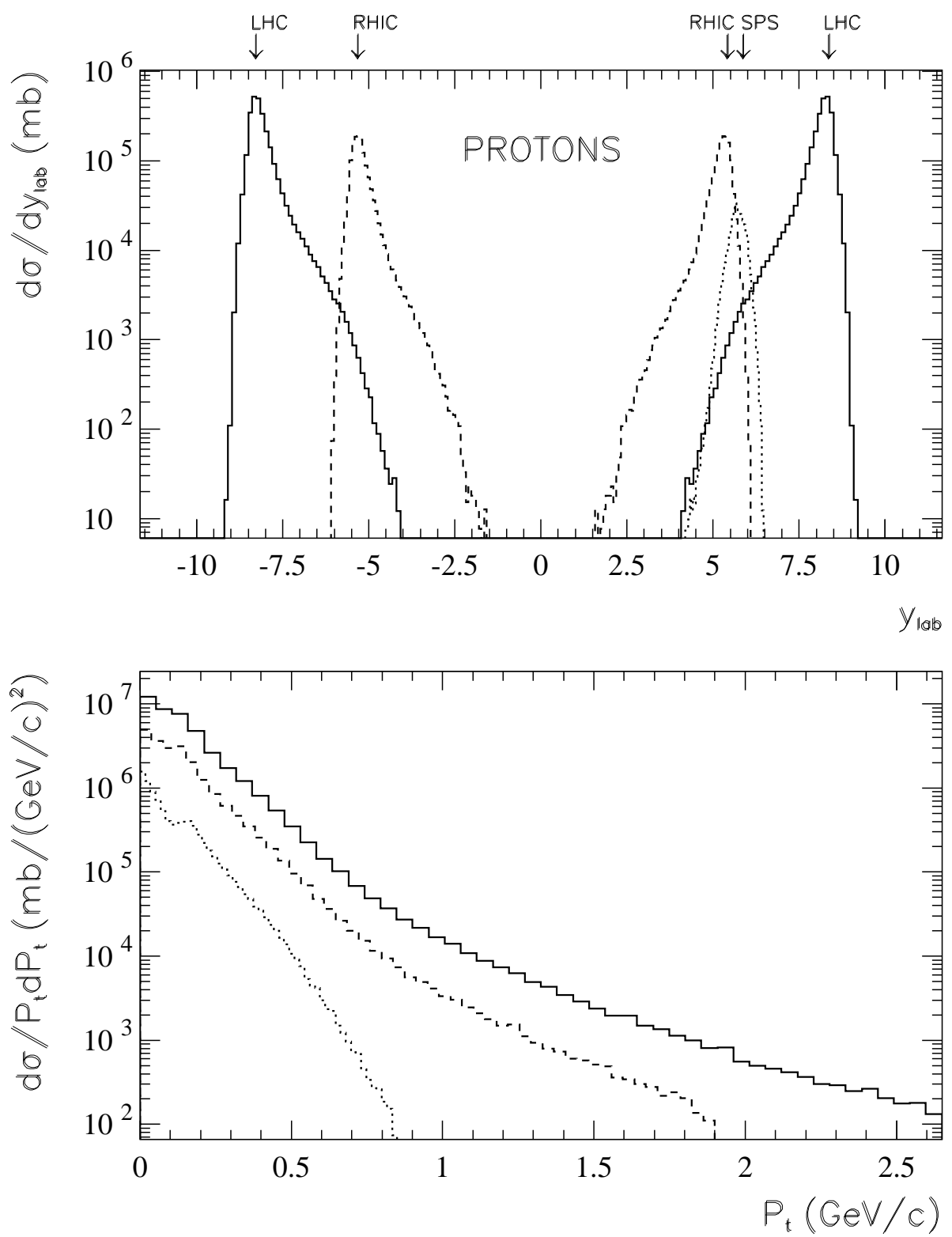

Figure 5: The same as in Fig. 1, but for protons. 


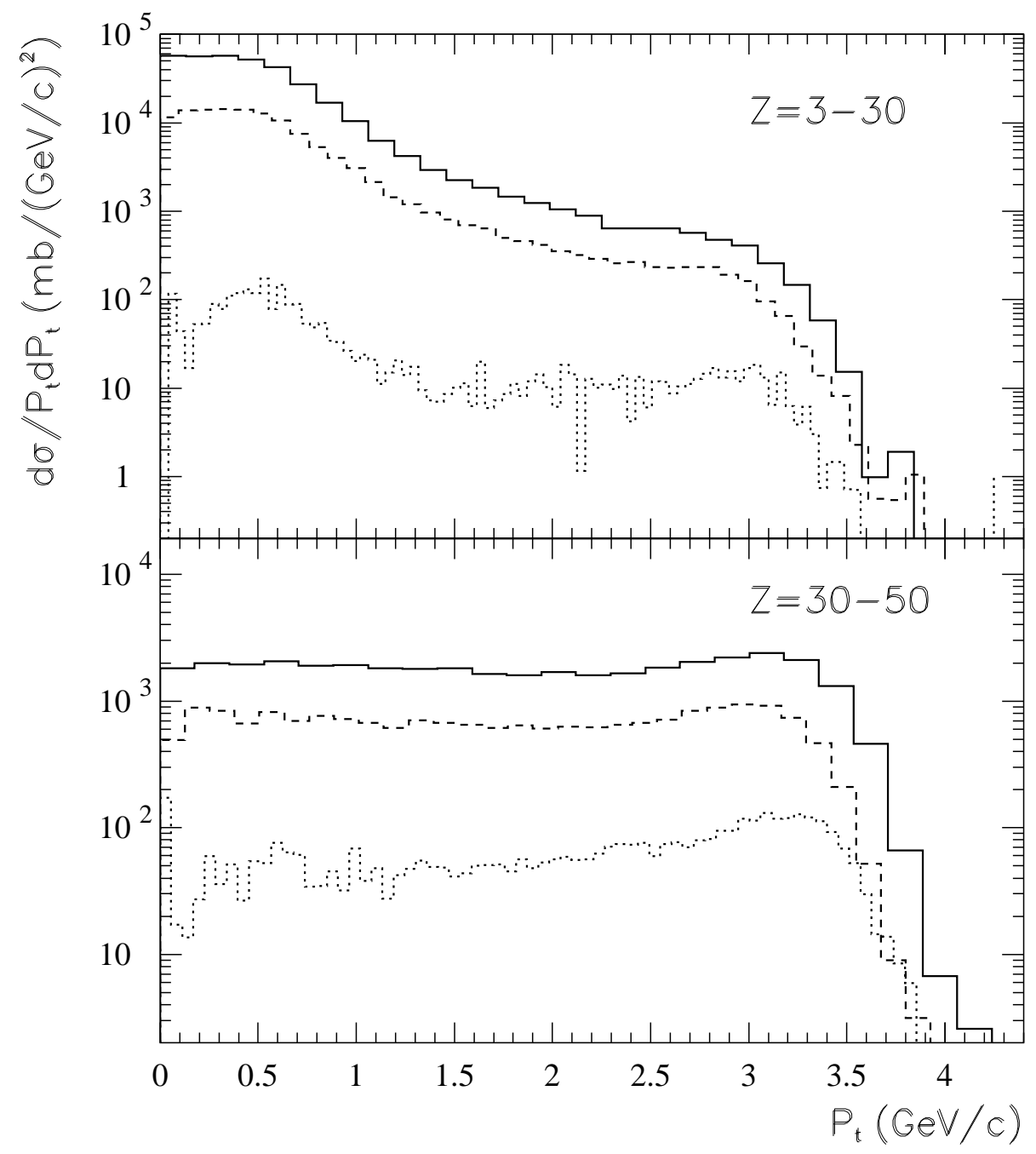

Figure 6: RELDIS predictions for inclusive transverse momentum distributions of intermediate mass (top panel) and fission (bottom panel) fragments emitted in the electromagnetic dissociation of Pb nuclei at LHC and SPS energies (solid and dotted lines, respectively) and Au nuclei at RHIC energies (dashed lines). 

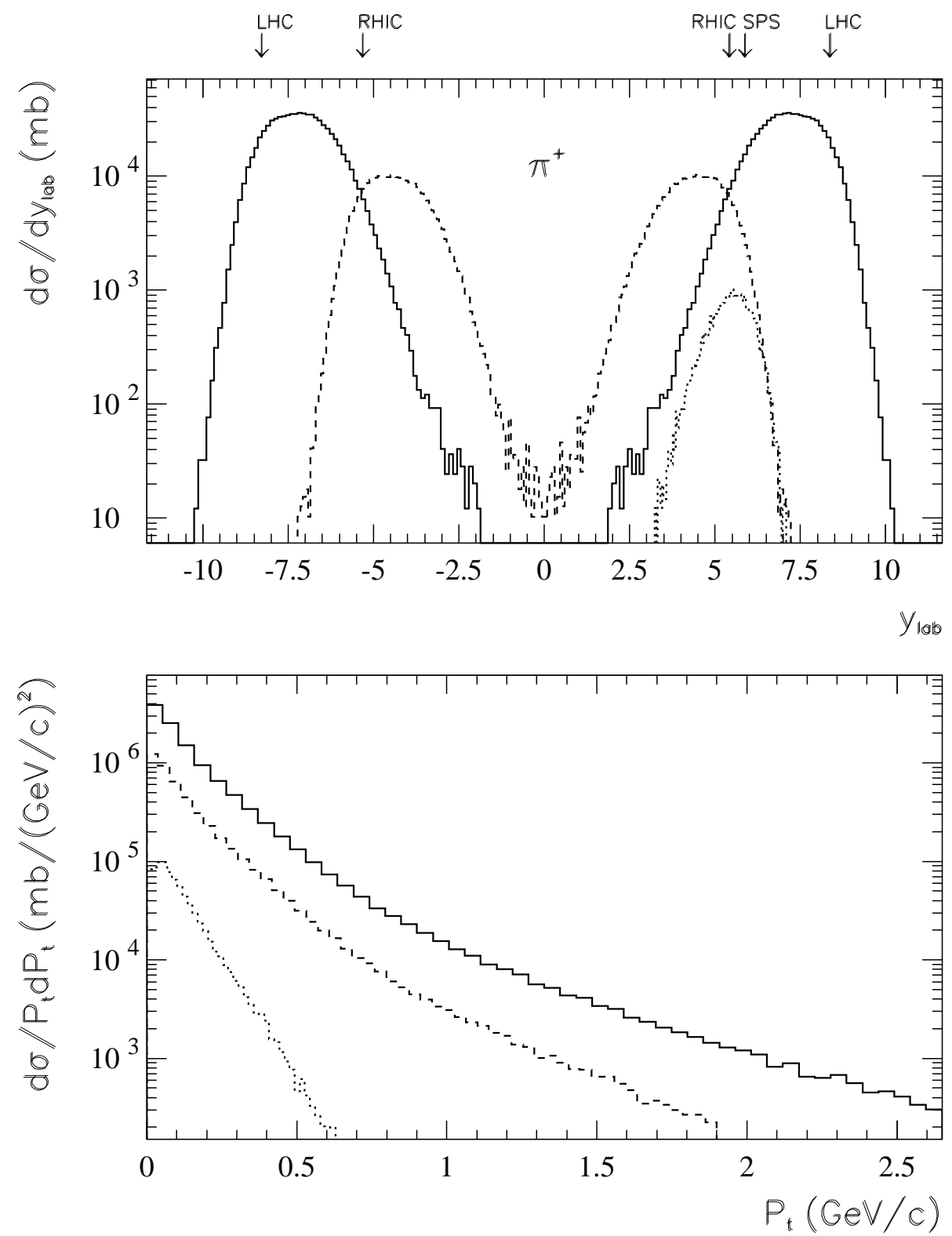

Figure 7: The same as in Fig. 团, but for positive pions. 

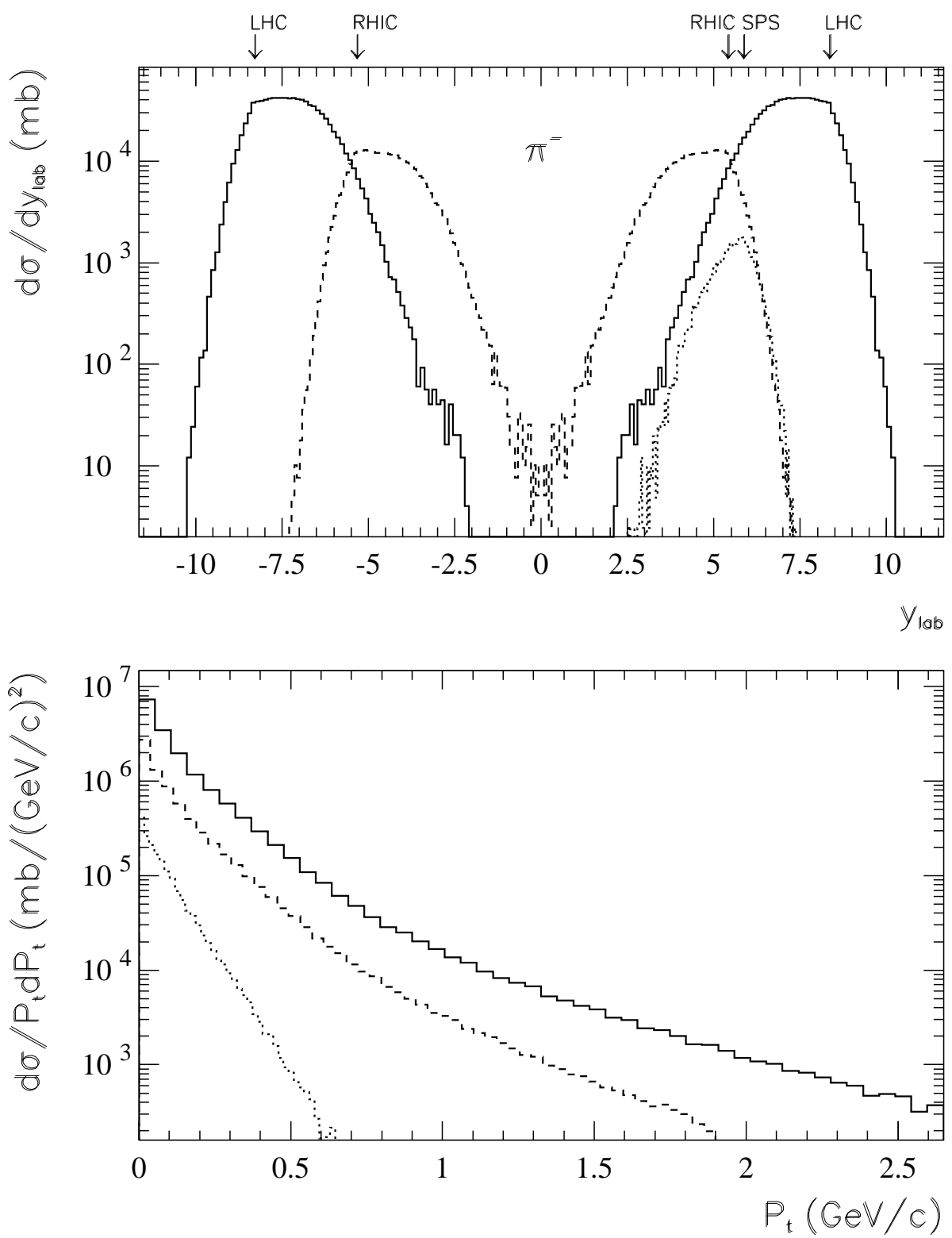

Figure 8: The same as in Fig. 1, but for negative pions. 